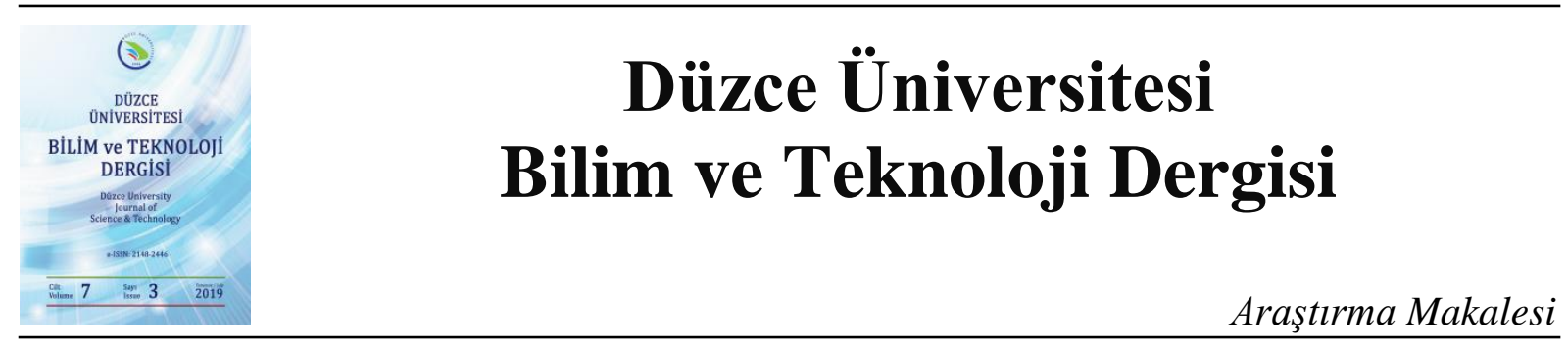

\title{
Düzce Şehir Ormanı Makrofungusları
}

\author{
(iD) Murat AKTAŞ ${ }^{\mathrm{a}}$, (D) Beşir YÜKSEL ${ }^{\mathrm{b}}$, (D) Nuray ÖZTÜRK ${ }^{\mathrm{b}}$, , iD Zehra KAÇAN $^{\mathrm{b}}$ \\ ${ }^{a}$ Mengen Orman İşletme Müdürlüğ̈̈, Bolu, TÜRKIYYE \\ ${ }^{b}$ Orman Mühendisliği Bölümü, Orman Fakültesi, Düzce Üniversitesi, Düzce, TÜRKIYE \\ * Sorumlu yazarın e-posta adresi: nurayozturk@duzce.edu.tr \\ DOI : 10.29130/dubited.527426
}

\begin{abstract}
ÖZET
Orman makrofungal toplumların çeşitliliği farklı işletme uygulamalarıyla yakından ilgilidir. Düzce Şehir Ormanı, 1974 yılından önce doğal meşe ormanı iken, sonra sahil çamı (Pinus pinaster) plantasyonu olarak tesis edilmiştir. Bu çalışma 2017 - 2018 yılları arasında Düzce Şehir Ormanından periyodik olarak toplanan makrofungus örnekleri üzerinde yapılmıştır. Arazi ve laboratuvar çalışmalarının sonucu olarak Basidiomycota bölümünde 28 familyaya ait 60 takson rapor edilmiştir. Tüm taksonlar habitat bilgileri, coğrafik konum, yükseklik, lokalite, etnomikolojik veriler, toplama tarihi ve numaraları ile birlikte listelenmiştir. Bunlardan 28'i yenilebilir, 20'si yenilemeyen ve 12 tanesi ise zehirli tür olarak değerlendirilmiştir. Belirlenen türlerden Lepiota cortinarius J. E. Lange ülkemiz için ilk kez kayıt edilmiştir.
\end{abstract}

Anahtar Kelimeler: Biyoçeşitlilik 1, Düzce 2, Makrofunguslar 3, Şehir Ormanı 4, Türkiye 5

\section{The Macrofungi Of Düzce Urban Forest}

\begin{abstract}
Diversity of forest macrofungal communities have been strongly influenced by different management approaches. While Düzce Urban Forest was a naturally oak forest in 1974, then it was converted to a pine plantation area by planting the maritime pine (Pinus pinaster). This study was based on the macrofungi species periodically collected from Düzce Urban Forest (Düzce) between 2017 - 2018. According to field and laboratory studies, 60 taxa belonging to 28 families of Basidiomycota division were reported. All taxa were listed together with their habitats, altitudes, localities, ethno-mycological data, collection dates, and accession numbers. Of this fungi species 28, 20 and 12 species were listed as edible, inedible and poisonous respectively. Among the identified macrofungi species, Lepiota cortinarius J. E. Lange was identified for the first time in Türkiye.
\end{abstract}

Keywords: Biodiversity 1, Düzce 2, Macrofungi 3, Turkey 4, Urban Forest 5

Geliş: 15/02/2019, Düzeltme: 18/03/2019, Kabul: 04/04/2019 


\section{GiRiș}

$\mathrm{M}^{\mathrm{s}}$ akrofungal çeşitlilik konusunda Türkiye'de çok sayıda çalışma yapılmış ve bunlar farklı zamanlarda kontrol listesi olarak yayınlanmıştır [1,2]. Araştırmaların sonucunda yeni kayıtlarla birlikte Türkiye'deki makrofungus listesine katkılar yapılmıştır [3]-[14]. Düzce Şehir Ormanının yakın çevresinde makrofungal çeşitlilik üzerine geçmiş yıllarda taksonomik çalışmalar gerçekleştirilmiştir [15]-[17]. Ancak bu şehir ormanında kapsamlı mikolojik çalışmalar yapılmamıştır. Özellikle sahil çamı plantasyonuyla meşcere kuruluşu değişen bu ormanlarda makrofungal çeşitliliğin ortaya konulması gerekmektedir. Bu çalışmanın amacı, Düzce Şehir Ormanının makrofungal toplumlarını belirlemek ve Türkiye makromikotasına katkı sağlamaktır.

\section{MATERYAL VE YÖNTEM}

Düzce Şehir Ormanında 2017-2018 yılları arasında yapılan arazi çalı̧masında sonbahar, ilkbahar ve yaz aylarında periyodik olarak makrofungus örnekleri toplanmıştır. Örneklerin habitat özellikleri kaydedildikten sonra fruktifikasyon organlarının özelliklerini gösteren fotoğrafları çekilmiştir. Bunların, tadı, kokusu ve renk değişimi gibi teşhiste kullanılabilecek ayırıcı özellikler arazi defterine işlenmiştir. Örnekler laboratuvara taşınarak, gerekli makroskopik ve mikroskopik ölçüm verileri elde edilmiştir. Veri toplama işlemlerinde bazı kimyasallar (distile su, $\mathrm{FeSO}_{4}, \mathrm{KOH}, \mathrm{NH}_{3}$, Fenol, Melzer ayıracı, Kongo kırmızısı, sulfo-vanilin vb.) kullanılarak, cins ve takson tanımları için değerlendirilmiştir. Örneklerin teşhisi literatür yardımı ile yapılmıştır [18]-[29]. Örnekler, Düzce Üniversitesi, Orman Fakültesi, Orman Entomoloji ve Koruma Anabilim Dalı Laboratuvarında tutulmaktadır. Düzce Şehir Ormanı Düzce ilinin kuzeydoğusunda yer almaktadır. Bu rekreasyonel kullanım alanının çevresinde Nalbantoğlu, Kazıkoğlu, Tokuşlar, Çakırlar, Çiftçiler, Esentepe köyleri ve Kalıcı konutlar yerleşim alanları bulunmaktadır. Ayrıca, Düzce-Zonguldak kuzey çevre yoluna sınır konumundadır (Şekil 1).

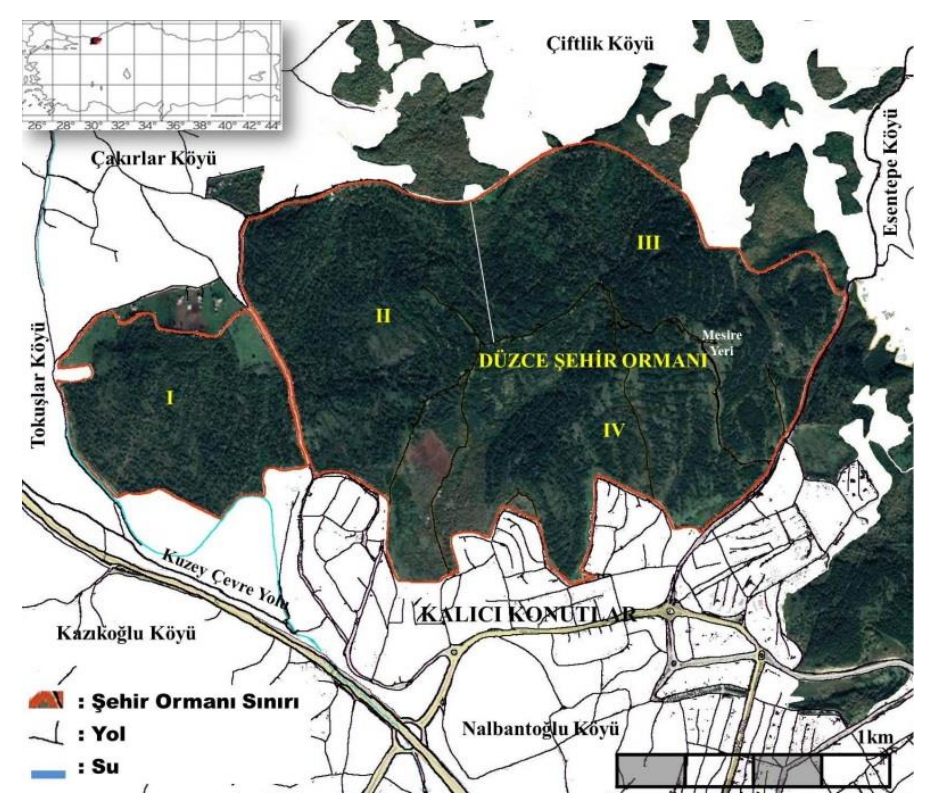

Şekil 1. Çalışma alanının haritası 
Bu alanın hakim vejetasyonu Pinus pinaster Ait. olup, yer yer Quercus frainetto Ten., Q. petrea subsp. iberica (Steven ex Bieb.) Krassilin., Q. pubescens Willd., Phillyrea latifolia L., Juniperus communis var. saxatilis Pall., Cretagus monogyna Jacq., Rubus hirtus Waldst. \& Kit., Arbutus unedo L., Paliurus spina-christi P. Mill., Pistacia terebinthus L., Mespilus germanica L., Cornus mas L., Hypericum calycinum L., Cistus creticus L., Rosa canina L. ve Ruscus aculeatus L. türleri bulunmaktadır. Düzce Merkez ilçe meteoroloji istasyonunun son üç y1llık iklim verilerine göre şehir ormanında en düşük sıcaklık ocak ayında $-12,0{ }^{\circ} \mathrm{C}$ ve en yüksek sicaklık ağustos ayında $42,2{ }^{\circ} \mathrm{C}$ 'dir. Yıllık yağış miktarı 917,67 mm'dir. Aylık ortalama bağıl nem miktarı en düşük \%67,9 ve en yüksek $\% 92,1$ olup ortalamas1 $\% 78,02$ 'dir.

\section{BULGULAR}

Düzce Şehir Ormanında alan ve laboratuvar çalışmaları sonucunda Basidiomycota'nın 28 familyasına ait 60 takson tanımlanmıştır. Taksonların sistematiğinde indexfungorum.org (erişim tarihi, 08 Ocak 2019) ve Kirk ve ark. [30] esas alınarak, yayılış, habitat, konum, toplanma tarihi ve laboratuvar numaralarıyla birlikte listelenmiştir.

Basidiomycota Whittaker ex Moore

Agaricales Underw.

Agaricaceae Chevall

Bovista aestivalis (Bonord.) Demoulin: P. pinaster, Q. pubescens ve Q. petraea çevresinde çayırlık,

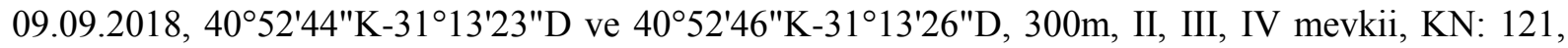
130, 131; Q. pubescens, J. communis ve mera alanında, 20.09.2018, 4052'36.86"K-31¹3'31"D, 190 210m, I mevkii, KN: 137.

Cyathus olla (Batsch) Pers.: devrik P. pinaster' in sürgün ve ince dallarında, 11.12.2017, 40 $52^{\prime} 41^{\prime \prime K}$ 31¹3'37"D, 332m, IV mevkii, KN: 44.

Cystolepiota seminuda (Lasch) Bon: P. pinaster altında otların içinde, 18.11.2017, 4052'25"K$31^{\circ} 13^{\prime} 48^{\prime \prime} \mathrm{D}, 277 \mathrm{~m}$, III mevkii, KN: 5.

Lepiota cortinarius J. E. Lange: $Q$. frainetto ve $P$. pinaster altında otların içinde, 18.11.2017, $40^{\circ} 52^{\prime} 23^{\prime \prime} \mathrm{K}-31^{\circ} 13^{\prime} 42^{\prime \prime} \mathrm{D}, 270 \mathrm{~m}$, III mevkii, KN: 6.

Şapka 3-6,8 cm çapında, hafif koni şeklinde, sarımsı-kahverengi, lameller beyazımsı ten renginde olup şapkanın kenar uçlarından saptan ayrılmış beyaz lifli ağımsı yapı görülür (Şekil 2a-b). Sap 4,0-7,4 x 0,6-1,1 cm boyutunda ve kaideye doğru daha kalın ve klavatl1-yumru görünümlüdür. Bazidyospor; 7.67-10.53 x 3.65-4.43 $\mu \mathrm{m}$, beyaz, porsuz, dekstrinoid ve penguen şeklindedir (Şekil 2c). $L$. cortinarius ile ilgili yapılan bazı çalışmalarda bazidyosporlarının 6,5-10 x 2,5-4,38 $\mu \mathrm{m}$ büyüklüğünde olduğu bildirilmektedir [20], [31]. Bazidyum; 18,0-26,5 x 6,0-8,5 $\mu \mathrm{m}$, tetra sporlu ve klavat şekillidir. Lamellerde büyük yapılı steril keliosistid'ler 6,84-8,00 x 20,96-26,92 $(-39,98) \mu \mathrm{m}$ boyutunda ve klavat formundadır (Şekil 2d). Trichodermanın içinde pileipellisler silindirik yapıda, az kıvrımlı olup, 80-225 x 7,8-10 $\mu$ m'dur. Bu türün görüldüğü Kasım ayının ortalama sıcaklığı $9,6{ }^{\circ} \mathrm{C}$ ve bağı nemi \% 84,2 'dir. 


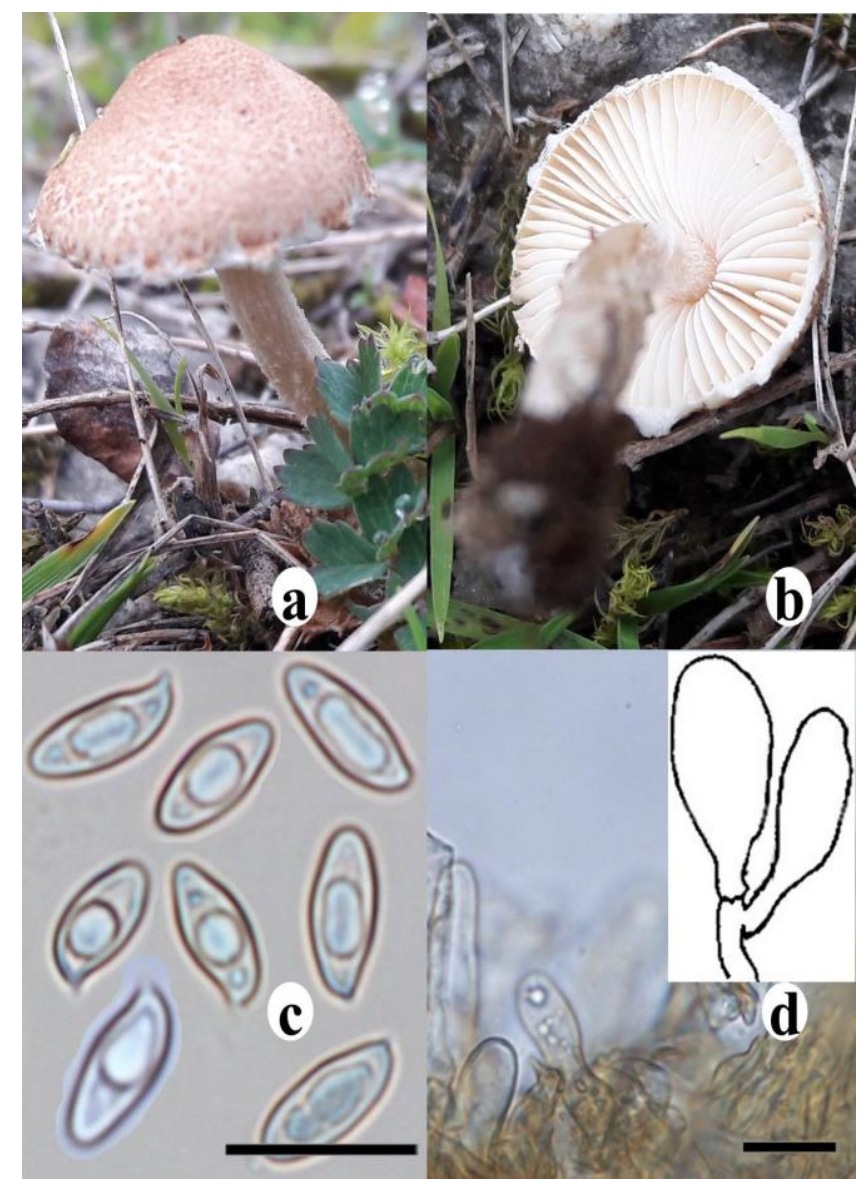

Şekil 2. Lepiota cortinarius; (a) ve (b) bazidyokarp, (c) bazidyospor, (d) keliosistid'ler (10 $\mu \mathrm{m})$

Lepiota echinella Quél \& G.E. Bernard: $Q$. frainetto ve P. pinaster altında otların içinde, 18.11.2017, $40^{\circ} 52^{\prime} 29^{\prime \prime K}-31^{\circ} 13^{\prime} 432 " \mathrm{D}, 292 \mathrm{~m}$, IV mevkii, KN: 10.

Lycoperdon lividum Pers.: P. pinaster, Q. pubescens ve Q. petraea, 12.08.2018, 40 52'42"K$31^{\circ} 13^{\prime} 39^{\prime \prime} \mathrm{D}, 320-334 \mathrm{~m}$, III mevkii, KN: 120.

Tulostoma brumale Pers.: $Q$. pubescens, $R$. canina ve $P$. latifolia türlerinin yanında yosun-çayır, 18.11.2017, 4052'21"K-31'13'50"D, 265m, IV mevkii, KN: 3.

Amanitaceae

Amanita crocea (Quel.) Singer: P. pinaster, Q. pubescens, Q. frainetto ve H. calycinum, 06.06.2018, $40^{\circ} 52^{\prime} 38^{\prime \prime K}-31^{\circ} 13 ' 38^{\prime \prime} \mathrm{D}, 40^{\circ} 52^{\prime} 24^{\prime \prime K}-31^{\circ} 13^{\prime} 44^{\prime \prime} \mathrm{D}, 270-330 \mathrm{~m}$, IV mevkii, KN: 103, 111.

Amanita argentea Huijsman: $P$. pinaster, $Q$. pubescens ve $H$. calycinum, 06.06.2018, 40 $52^{\prime} 39^{\prime \prime} \mathrm{K}-$ 31 ${ }^{\circ} 13^{\prime} 37^{\prime \prime} \mathrm{D}, 330 \mathrm{~m}$, IV mevkii, KN: 101.

Amanita vaginata var. alba (De Seynes) Gillet: $P$. pinaster, $Q$. pubescens ve $H$. calycinum, 06.06.2018, 40 $52^{\prime} 38^{\prime \prime} \mathrm{K}-31^{\circ} 13^{\prime} 38^{\prime \prime} \mathrm{D}, 330 \mathrm{~m}$, IV mevkii, KN: 96; P. pinaster, Q. petraea, $40^{\circ} 52^{\prime} 29^{\prime \prime} \mathrm{K}-$

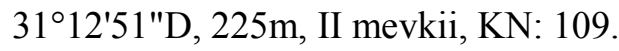

Entolomataceae Kotl. \& Pouzar 
Entoloma hirtipes (Schumach.) M.M. Moser: P. pinaster ve $Q$. frainetto altında yosun ve otlar

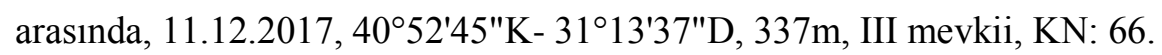

Entoloma sericeum Quél.: P. pinaster ve $Q$. frainetto'nun altında yosun ve otlar arasında, 11.12.2017, 405' $43^{\prime \prime K}-31^{\circ} 13^{\prime} 37^{\prime \prime} \mathrm{D}, 335 \mathrm{~m}$, III mevkii, KN: 72.

Hygrophoraceae Lotsy.

Cuphophyllus virgineus (Wulfen) Kovalenko: $P$. pinaster ve $Q$. frainetto altında, 18.11.2017, $40^{\circ} 52^{\prime} 25^{\prime \prime K}-31^{\circ} 13^{\prime} 26^{\prime \prime} \mathrm{D}, 260 \mathrm{~m}$, IV mevkii, KN: 14; $P$. pinaster altında yosun ve otlar içinde, 18.11.2017, 4052'26"K-31¹3'45"D, 280m, IV mevkii, KN: 33

Hygrocybe acutoconica (Clem.) Singer: P. pinaster, $R$. canina ve $P$. latifolia türlerinin yanında çayırmera, 18.11.2017, 40 $52^{\prime} 24^{\prime \prime K}-31^{\circ} 13^{\prime} 49^{\prime \prime} \mathrm{D}, 260-270 \mathrm{~m}$, IV mevkii, KN: 1; 40 $52^{\prime} 26^{\prime \prime K}-31^{\circ} 13^{\prime} 52^{\prime \prime} \mathrm{D}$, 271m, III mevkii, KN: 8; 405' $26^{\circ} \mathrm{K}-31^{\circ} 13^{\prime} 45^{\prime \prime} \mathrm{D}, 280 \mathrm{~m}$, IV mevkii, KN: 32.

Hydnangiaceae Gäum. \& C.W. Dodge

Laccaria amethystina Cooke: P. pinaster ve $Q$. petraea altında, $18.11 .2017,40^{\circ} 52^{\prime} 29^{\prime \prime} \mathrm{K}-31^{\circ} 13^{\prime} 26^{\prime \prime} \mathrm{D}$, 280m, IV mevkii, KN: 27.

Laccaria laccata (Scop.) Cooke: $P$. pinaster, $Q$. frainetto ve $P$. latifolia altinda yosun ve otlarla birlikte, 18.11.2017, 4052'45"K-31'13'36"D, 336m, IV mevkii, KN: 27.

Hymenogastraceae Vittad.

Hebeloma album Peck.: P. pinaster ve $Q$. frainetto altında yosunlu alanda, $11.12 .2017,40^{\circ} 52^{\prime} 45^{\prime \prime} \mathrm{K}$ 31 13 '37"D, 336m, III mevkii, KN: 71.

Inocybaceae Jülich

Crepidotus cesatii (Rabenh.) Sacc.: Q. frainetto'un kütüğünde 11.12.2017, 4052'41.66"K$31^{\circ} 13^{\prime} 40.29 " \mathrm{D}, 332 \mathrm{~m}$, III mevkii, KN: 38.

Inocybe assimilata Britzelm.: $Q$. frainetto ve $P$. pinaster altında, 18.11.2017, 40 $52^{\prime} 29^{\prime \prime} \mathrm{K}$ -

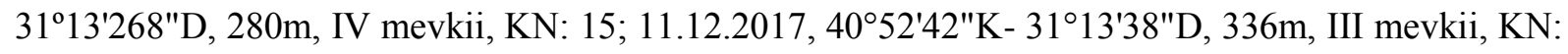
59; Q. frainetto ve P. latifolia altında, 11.12.2017, 40 $52^{\prime} 44^{\prime \prime K}-31^{\circ} 13 ' 37 " \mathrm{D}, 325-337 \mathrm{~m}$, III mevkii, $\mathrm{KN}: 41,63,70$.

Inocybe cincinnata (Fr.) Quél.: Q. frainetto ve P. latifolia altında, 11.12.2017, 4052'44"K31 ${ }^{\circ} 13^{\prime} 37^{\prime \prime} \mathrm{D}, 337 \mathrm{~m}$, III mevkii, KN: 62.

Inocybe fuscidula Velen.: $Q$. frainetto ve $P$. pinaster altında liken ve otların içinde, 18.11.2017, $40^{\circ} 52^{\prime} 22^{\prime \prime} \mathrm{K}-31^{\circ} 13^{\prime} 48^{\prime \prime} \mathrm{D}, 265 \mathrm{~m}$, IV mevkii, KN: 10.

Inocybe geophylla (Pers.) P. Kumm.: Q. frainetto ve P. pinaster altında, 11.12.2017, 40 $52^{\prime} 43^{\prime \prime} \mathrm{K}-$ 31¹3'38"D, 336m, III mevkii, KN: 60.

Inocybe queletii Konrad: $Q$. frainetto, $P$. pinaster ve P. latifolia altında, $11.12 .2017,40^{\circ} 52^{\prime} 42^{\prime \prime} \mathrm{K}$ 3113'38"D, 334-337m, III mevkii, KN: 59, 65, 66.

Marasmiaceae Roze ex Kühner 
Marasmius oreades (Bolton) Fr.: P. pinaster, Q. pubescens ve $Q$. petraea altında otlar arasinda, 20.09.2018, 4052'30"K-31 ${ }^{\circ} 12^{\prime} 33^{\prime \prime} \mathrm{D}$ ve $40^{\circ} 52^{\prime} 30^{\prime \prime K}-31^{\circ} 39^{\prime \prime} \mathrm{D}, 180-190 \mathrm{~m}$, I, II mevkii, KN: 134, 149; $P$. pinaster ve $Q$. frainetto altında, $26.09 .2018,40^{\circ} 52^{\prime} 27^{\prime \prime K}-31^{\circ} 13^{\prime} 50^{\prime \prime} \mathrm{D}$ ve $40^{\circ} 52^{\prime} 24^{\prime \prime} \mathrm{K}-31^{\circ} 13^{\prime} 42^{\prime \prime} \mathrm{D}$, 270-280m, III, IV mevkii, KN: 150, 151; $P$. pinaster ve $Q$. frainetto altında, 05.10.2018, 40 $52^{\prime 2} 27^{\prime \prime} \mathrm{K}-$ $31^{\circ} 13^{\prime} 47^{\prime \prime} \mathrm{D}, 280 \mathrm{~m}$, III mevkii, KN: 158.

Omphalotaceae Bresinsky

Mycetinis scorodonius (Fr.) A.W. Wilson \& Desjardin: P. pinaster, Q. pubescens ve $Q$. petraea altında, $09.09 .2018,40^{\circ} 52^{\prime} 44^{\prime \prime} \mathrm{K}-31^{\circ} 13^{\prime} 23^{\prime \prime} \mathrm{D}$ ve $40^{\circ} 52^{\prime} 46^{\prime \prime} \mathrm{K}-31^{\circ} 13^{\prime} 26^{\prime \prime} \mathrm{D}, 300 \mathrm{~m}$, II, III, IV mevkii, KN: 123, 132, 133.

Schizophyllaceae Quél.

Schizophyllum commune Fr.: $Q$. frainetto'un dal ve kütügünde 11.12.2017, 4052'41.66"K$31^{\circ} 13^{\prime} 40.29^{\prime \prime} \mathrm{D}, 332 \mathrm{~m}$, III mevkii, KN: 37, 39; devrik $P$. pinaster' in kabuklu dal ve kütügünde, 11.12.2017, 40 $52^{\prime} 41.24^{\prime \prime} \mathrm{K}-31^{\circ} 13^{\prime} 37.63^{\prime \prime} \mathrm{D}, 332 \mathrm{~m}$, IV mevkii, $\mathrm{KN}$ : 50; kar kırmas1 P. pinaster'in dallarında, 05.10.2018, 40 $52^{\prime} 27^{\prime \prime K}-31^{\circ} 13^{\prime} 48$ "D, 280m, III mevkii, KN: 164.

Tricholomataceae R. Hein Ex Pouzar

Clitocybe amarescens Harmaja: P. pinaster altında yosun ve otlar arasında, 18.11.2017, 40 52'25"K$31^{\circ} 13$ '25"D, 265m, III mevkii, KN: 11.

Tricholoma equestre (L.) P. Kumm.: P. pinaster ve $Q$. pubescens altında, 18.11.2017, 4052'27"K$31^{\circ} 13^{\prime} 48^{\prime \prime} \mathrm{D}, 240-290 \mathrm{~m}$, III-IV mevkii, KN: 22, 26, 29

Tricholoma sejunctum (Sowerby) Quél.: $P$. pinaster ve $Q$. frainetto altında, 18.11.2017, $40^{\circ} 52^{\prime} 22.40^{\prime \prime} \mathrm{K}-31^{\circ} 13^{\prime} 50^{\prime \prime} \mathrm{D}, 265 \mathrm{~m}$, IV mevkii, $\mathrm{KN}$ : 12 ; $P$. pinaster ve $Q$. pubescens altında,

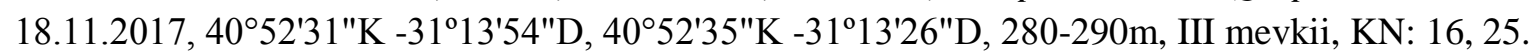

Tricholoma terreum (Schaeff.) P. Kumm.: P. pinaster altında, 18.11.2017, 40 52 '23"K-31 $13^{\circ} 48^{\prime \prime} \mathrm{D}$, 280m, IV mevkii, KN: 7.

\section{Auriculariales}

Auriculariaceae Fr.

Exidia glandulosa (Bull.) Fr.: P. pinaster' in kütüğünde, 11.12.2017, 40 $52^{\prime} 40^{\prime \prime K}-31^{\circ} 13^{\prime} 36^{\prime \prime} \mathrm{D}, 330 \mathrm{~m}$, IV mevkii, KN: 45.

Exidia recisa (Ditmar) Fr.: Q. frainetto'nun kesilmiş dalları üstünde, 11.12.2017, 4052'22.48"K-

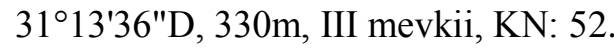

Boletales E.-J. Gilbert

Diplocystidiaceae Kreisel

Astraeus hygrometricus (Pers.) Morgan: P. pinaster, Q. pubescens ve $Q$. petraea'nin altında yosunlu alanda, 11.12.2017, 40 $52^{\prime} 45.48^{\prime \prime K}-31^{\circ} 13^{\prime} 38^{\prime \prime} \mathrm{D}, 340 \mathrm{~m}$, III mevkii, KN: 89.

Rhizopogonaceae Gäum. \& C.W. Dodge 
Rhizopogon ochraceorubens A.H. Sm.: P. pinaster, $Q$. pubescens, $Q$. petraea ve $P$. latifolia'l patika yolda toprakta, 11.12.2017, 40 $52^{\prime} 42^{\prime \prime} \mathrm{K}-31^{\circ} 13^{\prime} 38^{\prime \prime} \mathrm{D}, 334-338 \mathrm{~m}$, III mevkii, KN: 54, 56, 61.

Suillaceae Besl \& Bresinsky

Suillus collinitus (Fr.) Kuntze: P. pinaster ve Q. pubescens' in altında otlar arasinda, 11.12.2017, $40^{\circ} 52^{\prime} 40.28^{\prime \prime} \mathrm{K}-31^{\circ} 13^{\prime} 37.42^{\prime \prime} \mathrm{D}, 332 \mathrm{~m}$, IV mevkii, KN: 46; $P$. pinaster ve $Q$. pubescens, 06.06.2018, $40^{\circ} 52^{\prime} 41^{\prime \prime K}-31^{\circ} 13^{\prime} 38^{\prime \prime} \mathrm{D}, 334 \mathrm{~m}$, IV mevkii, KN: 98.

Cantharellales Gäum.

Cantharellaceae J. Schröt.

Cantharellus cinereus (Pers.) Fr.: P. pinaster ve çok az Q. pubescens altında çayır-mera, 18.11.2017, $40^{\circ} 52^{\prime} 21^{\prime \prime K}-31^{\circ} 13^{\prime} 47^{\prime \prime} \mathrm{D}, 262 \mathrm{~m}$, IV mevkii, KN: 2.

Clavulinaceae Donk

Clavulina cinerea (Bull.) J. Schröt.: P. pinaster, Q. pubescens ve $Q$. petraea'nin altında, 11.12.2017,

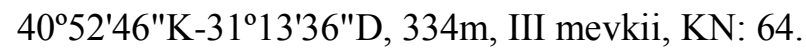

Polyporales Gäum.

Fomitopsidaceae Jülich

Daedalea quercina (L.) Pers.: Q. sp.'nin kütüğünde, 06.06 .2018 ve $12.08 .2018,40^{\circ} 52^{\prime} 33$ "K$31^{\circ} 13^{\prime} 42^{\prime \prime} \mathrm{D}, 300 \mathrm{~m}$, IV mevkii, KN: 105, 119.

Phaeolus schweinitzii (Fr.) Pat.: P. pinaster'in kırılmış gövdesinde, 05.10.2018, 4052'27"K$31^{\circ} 13^{\prime} 47^{\prime \prime} \mathrm{D}, 280 \mathrm{~m}$, III mevkii, KN: 152.

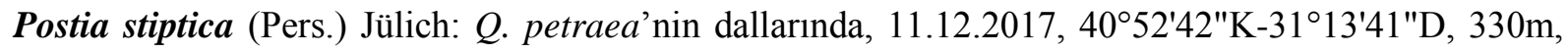
III mevkii, KN: 162.

Ganodermataceae Donk

Ganoderma resinaceum Boud.: Q. pubescens'in gövdesinde, 20.09.2018, 4052'36.86"K-31'13'31"D, 199m, I mevkii, KN: 136.

Meruliaceae Rea

Steccherinum ochraceum (Pers.) Gray: Q. pubescens'in kesilmiş dallarında, 11.12.2017, $40^{\circ} 52^{\prime} 45.46^{\prime \prime} \mathrm{K}-31^{\circ} 13^{\prime} 35^{\prime \prime} \mathrm{D}, 330 \mathrm{~m}$, III mevkii, KN: 74.

Phanerochaetaceae Jülich

Byssomerulius corium (Pers.) Parmasto: $Q$. pubescens ve $Q$. petraea'nin kurumuş dallarında, 12.08.2018, 40 $52^{\prime} 45^{\prime \prime} \mathrm{K}-31^{\circ} 13^{\prime} 37^{\prime \prime} \mathrm{D}, 334-338 \mathrm{~m}$, III mevkii, KN: 118; devrik $Q$. pubescens ve $Q$. petraea dallarında, 09.09.2018, 4052'44"K-31'13'26"D, 300m, IV mevkii, KN: 124.

Polyporaceae Fr. ex Corda

Faerberia carbonaria (Alb. \& Schwein.) Pouzar: P. pinaster ve $Q$. frainetto altında yosun ve otlar arasında, 05.10.2018, 4052'26"K-31'13'46"D, 290m, III mevkii, KN: 159. 
Hapalopilus nidulans (Fr. : Fr.) P. Karst.: P. pinaster'in kütüğünde, 18.11.2017, 4052'30"K$31^{\circ} 13^{\prime} 43^{\prime \prime} \mathrm{D}, 310 \mathrm{~m}$, IV mevkii, $\mathrm{KN}: 30 ; P$. pinaster'in kütüğ̈nde, $11.06 .2018,40^{\circ} 52^{\prime 2} 28^{\prime \prime} \mathrm{K}-$ $31^{\circ} 12^{\prime} 06^{\prime \prime} \mathrm{D}, 200 \mathrm{~m}$, IV mevkii, KN: 112.

Trametes hirsuta (Wulfen) Lloyd: Q. petraea'nin dallarında, 11.12.2017, 4052'42"K-31'13'41"D, 330m, III mevkii, KN: 163.

Trametes versicolor (L.) Lloyd: $Q$. pubescens ve $Q$. petraea'nin kesilmiş eski kütüklerinde, 11.12.2017 ve $09.09 .2018,40^{\circ} 52^{\prime} 42^{\prime \prime} \mathrm{K}-31^{\circ} 133^{\prime} 38^{\prime \prime} \mathrm{D}, 330-237 \mathrm{~m}$, III mevkii, KN: 78, 126; Q. pubescens ve $Q$. petraea'nin kütük ve dalarında, $12.08 .2018,40^{\circ} 52^{\prime} 45^{\prime \prime K}-31^{\circ} 13 ' 38^{\prime \prime} \mathrm{D}, 340 \mathrm{~m}$, III mevkii, KN: 114, 115; Q. petraea devriği altında kalan P. latifolia'nın kuru gövdesinde, 20.09.2018, 4052'30"K$31^{\circ} 12^{\prime 2} 28^{\prime \prime} \mathrm{D}, 200 \mathrm{~m}$, I mevkii, KN: 144; kar kırmas1 $P$. pinaster'in kabuklu gövdesinde, 05.10.2018, $40^{\circ} 52^{\prime} 32^{\prime \prime} \mathrm{K}-31^{\circ} 13^{\prime} 41^{\prime \prime} \mathrm{D}, 305 \mathrm{~m}$, IV mevkii, KN: 165.

Russulales Kreisel ex P.M. Kirk, P.F. Cannon \& J.C. David Albatrellaceae Nuss

Laeticutis cristata (Schaeff.) Audet: kesilmiş eski Q. petraea'nin gövdesi altında toprakta, 11.12.2017, 4052'42"K-31ํ'13'41"D, 330m, III mevkii, KN: 43.

Bondarzewiaceae Kotl. \& Pouzar

Bondarzewia mesenterica (Schaeff.) Kreisel: yosun ve otların kapladığı $Q$. sp. kütüğünde, 12.08.2018, 405'ㄴ' $42^{\prime \prime}-31^{\circ} 13^{\prime} 38^{\prime \prime} \mathrm{D}, 334 \mathrm{~m}$, III mevkii, KN: 113.

Russulaceae Lotsy

Lactarius azonites (Bull.) Fr.: P. pinaster ve $Q$. frainetto altında, 05.10.2018, 4052'27"K-31 $133^{\prime} 47^{\prime \prime} \mathrm{D}$, 280m, III mevkii, KN: 157.

Lactarius deliciosus (L.) Gray: $P$. pinaster ve $Q$. frainetto altında, 18.11.2017, 40 $52^{\prime} 23^{\prime \prime} \mathrm{K}$ $31^{\circ} 13^{\prime} 45^{\prime \prime} \mathrm{D}, 275 \mathrm{~m}$, IV mevkii, KN: 13. $P$. pinaster, $Q$. pubescens ve $Q$. petraea ve $P$. latifolia, 06.06.2018, 40 $52^{\prime} 42^{\prime \prime} \mathrm{K}-31^{\circ} 13^{\prime} 37^{\prime \prime} \mathrm{D}, 334 \mathrm{~m}$, III mevkii, KN: 95; P. pinaster, $Q$. pubescens ve $H$.

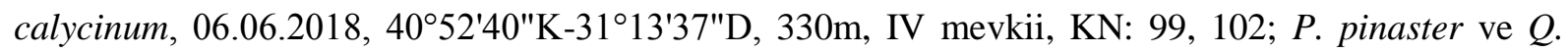
frainetto altında, $06.06 .2018,40^{\circ} 52^{\prime} 29^{\prime \prime K}-31^{\circ} 13 ' 39.48^{\prime \prime} \mathrm{D}, 290 \mathrm{~m}$, IV mevkii, $\mathrm{KN}$ : 107 ; $P$. pinaster ve Q. petraea, 06.06.2018, 40³1'42"K-31²12'43"D, 206m, II mevkii, KN: 108.

Lactarius piperatus (L.) Pers.: P. pinaster ve $Q$. frainetto altında, 06.06.2018, $40^{\circ} 52^{\prime} 41^{\prime \prime} \mathrm{K}-$

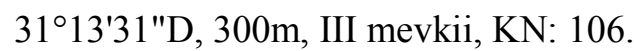

Russula erythropus Fr. ex Pelt.: P. pinaster ve $Q$. pubescens'in altında, 06.06.2018, 40 $52^{\prime} 37^{\prime \prime} \mathrm{K}$ 31¹3'38"D, 325m, IV mevkii, KN: 104.

Russula heterophylla (Fr.) Fr.: P. pinaster, Q. pubescens, 06.06.2018, 40 $52^{\prime} 40^{\prime \prime} \mathrm{K}-31^{\circ} 13^{\prime} 37^{\prime \prime} \mathrm{D}, 260$ $330 \mathrm{~m}$, IV mevkii, KN: 100; P. pinaster ve $Q$. petraea, $06.06 .2018,40^{\circ} 52^{\prime} 38^{\prime \prime} \mathrm{K}-31^{\circ} 12^{\prime} 53^{\prime \prime} \mathrm{D}, 210$ 270m, II mevkii, KN: 110; $P$. pinaster'in altında, 05.10.2018, 40 $52 ' 29^{\prime \prime} \mathrm{K}-31^{\circ} 13^{\prime} 41^{\prime \prime} \mathrm{D}, 290 \mathrm{~m}$, IV mevkii, KN: 156. 
Russula queletii Fr.: $P$. pinaster, $Q$. pubescens, M. germanica, C. monogyna ve $P$. latifolia, 06.06.2018, 4052'41"K-31²13'37"D, 333m, IV mevkii, KN: 97.

Russula rosea Pers.: P. pinaster altında yosun ve otlar içinde, $18.11 .2017,40^{\circ} 52^{\prime} 30^{\prime \prime} \mathrm{K}-31^{\circ} 13^{\prime} 43^{\prime \prime} \mathrm{D}$,

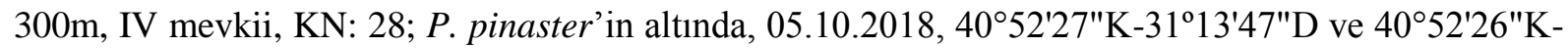
$31^{\circ} 13^{\prime} 42^{\prime \prime} \mathrm{D}, 280 \mathrm{~m}$, III, IV mevkii, KN: 153, 161.

Russula violeipes Quél.: P. pinaster'in altında, 05.10.2018, 4052'26"K-31'13'42"D, 280m, IV mevkii, KN: 154, 155.

Stereaceae Pilát

Stereum hirsutum (Willd.) Pers.: Q. petraea'nin eski kütügü üstünde, 11.12.2017, 4052'22.42"K$31^{\circ} 13^{\prime} 38^{\prime \prime} \mathrm{D}, 335 \mathrm{~m}$, III mevkii, KN: 57; Q. pubescens ve $Q$. petraea'nin kütüklerinde, 12.08.2018,

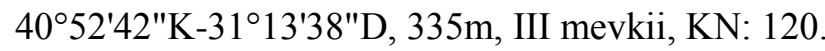

Thelephorales Corner ex Oberw.

Bankeraceae Donk

Hydnellum concrescens (Pers.) Banker: Q. pubescens ve $Q$. petraea'nin kesilmiş kütüklerinin olduğu çayırlık, 11.12.2017, 4052'41.68"K-31'13'38,50"D, 333m, III mevkii, KN: 77.

\section{Tremellales}

Tremellaceae Fr.

Tremella mesenterica Retz:: $Q$. pubescens ve $Q$. petraea'nin kesilmiş dallarında, 11.12.2017, $40^{\circ} 52^{\prime} 46^{\prime \prime K}-31^{\circ} 13^{\prime} 35^{\prime \prime} \mathrm{D}, 40^{\circ} 52^{\prime} 42^{\prime \prime K}-31^{\circ} 13^{\prime} 44^{\prime \prime} \mathrm{D}, 320-325 \mathrm{~m}$, III mevkii, KN: 42, 74; P. pinaster'in

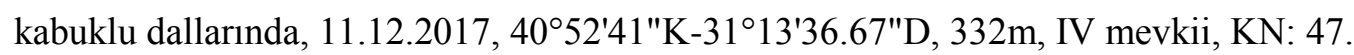

\section{TARTIȘMA VE SONUC}

Bu çalışmada Basidiomycota bölümünden 28 familya ve 41 cinse ait 60 takson ortaya konulmuştur. Taksonların familyalara dağılımı Şekil 3'te görülmektedir. Bu familyalar arasında Agaricaceae ve Russulaceae'nin 8'er taksonla temsil edilmektedir. Belirlenen taksonlardan Lepiota cortinarius Türkiye için yeni kayıttır. 


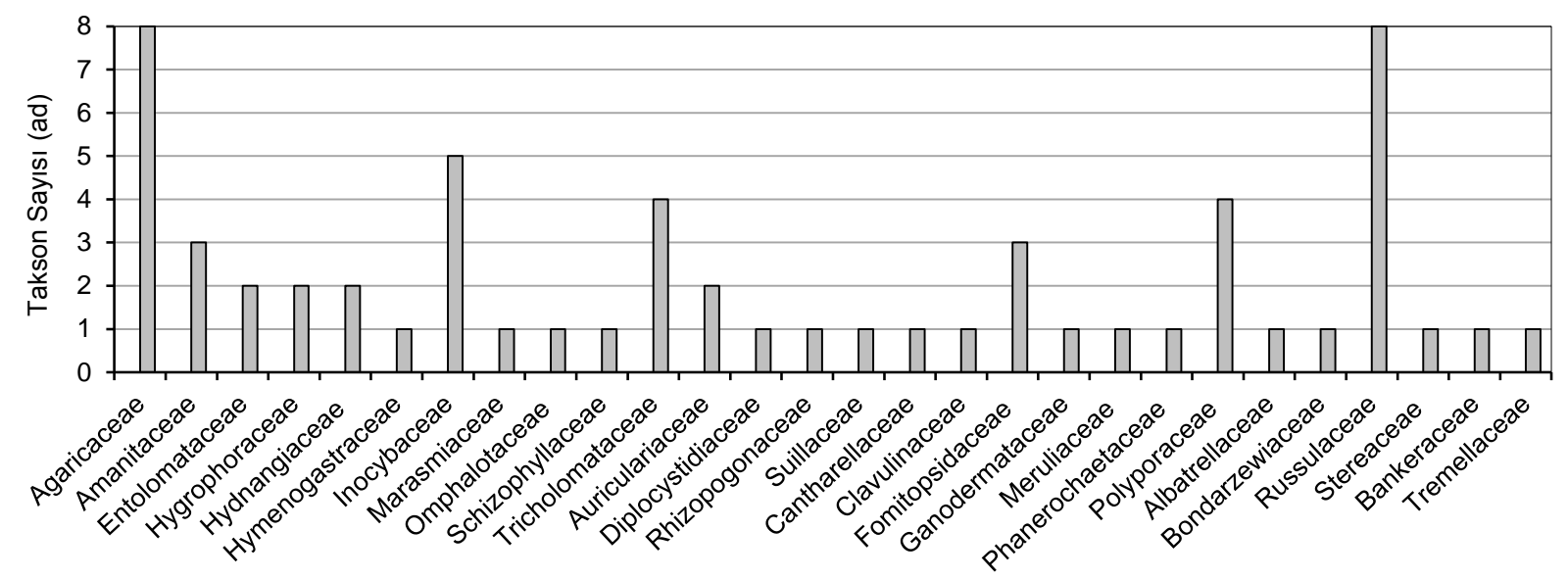

Şekil 3. Taksonların familyalara dağılımı

Tanımlanan taksonların 28 adedi yenilebilir, 20 adedi yenilemez ve 12 adedi ise zehirli olarak sınıflandırılmıştır (Şekil 4). Belirlenen örneklerin büyük bölümü yenilebilir özellikte olup, bu türlerden L. deliciosus'un “melki”, T. terreum'un “'Karagörece”, L. piperatus'un "acı mantarı" ve $M$. oreades'in de "mıhbaşı" veya "cincile" adıyla yöre halkı tarafından tanındığı ve pazar ürünü olarak değerlendirildiği görülmüştür. Yılmaz ve Zencirci [32]' e göre yöre halkı Batı Karadeniz Bölgesinde 33 taksonu yöresel isimleriyle toplayarak tüketmektedir. Bu yüzden, şehir ormanında doğal olarak yetişmekte olan mantarların halka tanıtılarak besin değeri olan türlerin ticaretinin teşvik edilmesi yöre halkına ekonomik olarak katkı sağlayacaktır.

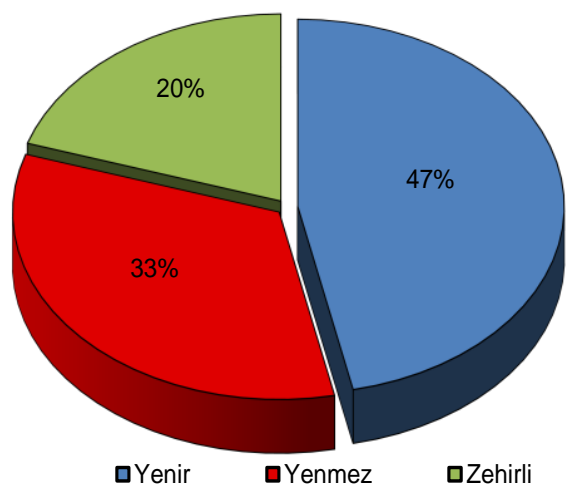

Şekil 4. Taksonların yenilebilirliği

Düzce Şehir Ormanında ayrıca 12 farklı zehirli mantar türü tanımlanmıştır. Bunlar: C. seminuda, $L$. cortinarius, L. echinella, E. hirtipes, E. sericeum, I. assimilata, I. cincinnata, I. fuscidula, I. geophylla, I. queletii, C. amarescens ve T. sejunctum taksonlarıdır. Çalışma yöresinde herhangi bir zehirlenme vakası bilinmemektedir. Bunun nedeni, yöre halkının sadece iyi bilinen yenilebilir mantarları toplaması ve tüketmesidir. Bu bağlamda, yörede yetişmekte olan mantarların halka tanıtılarak besin değeri olan türlerin ticaretinin teşvik edilmesi yöreye ekonomik olarak katkı sağlayacaktır.

Basidiomycota bölümüyle ilgili olarak, araştırma alanına yakın çevre ve komşu bölgelerde yapılan çalışmaların bulgularıyla mevcut çalışma arasında \%7,84-21,52 düzeyinde (Tablo 1) benzerlik bulunmaktadır [3], [15], [16], [33]-[39]. Tablo 1'e göre Kocaeli yöresi için takson sayıları ve ilgili çalışmalar arasındaki benzerlik \%21,52, Bartın-Küre Dağları Milli Parkı bölümü için \%16,92 [36] olup yakın çevresi Bolu ve Düzce yöresinde 26 ortak taksonla en yüksek sayıya ulaşılmasına rağmen 
benzerlik oranı \%10'dur [16]. Düzce Şehir Ormanı, makrofungal toplumların çeşitliliği yönünden benzerlik oranlarının düşük olması, özellikle yapay sahil çamı plantasyonuyla hakim bitki türünün tesisi ve buna yönelik farklı işletme uygulamalarının bir sonucu olarak değerlendirilebilir. Gassibe ve ark. [40]'e göre bu durum, ekstrem ekolojik koşullarda bulunan $P$. pinaster'in meşcerelerinin yüksek fungal potansiyele ve çeşitliliğe sahip olmasının bir sonucudur.

Tablo 1. Bölgeye yakın çalışmaların mevcut çalışmayla karşılaştırılması

\begin{tabular}{lccc}
\hline \multicolumn{1}{c}{ Yakın Bölgesel Çalışmalar } & $\begin{array}{c}\text { Tanımlanan Ortak } \\
\text { Takson Sayısı (adet) }\end{array}$ & $\begin{array}{c}\text { Toplam } \\
\text { Takson (adet) }\end{array}$ & $\begin{array}{c}\text { Benzerlik } \\
\text { Oranları (\%) }\end{array}$ \\
\hline $\begin{array}{l}\text { Bolu-Şerif Yüksel Araştırma } \\
\text { Ormanı [37] }\end{array}$ & 4 & 51 & 7,84 \\
\hline Bolu-Abant [34] & 13 & 98 & 13,27 \\
\hline Batı Karadeniz Bölgesi [15] & 13 & 94 & 13,83 \\
\hline Batı Karadeniz Bölgesi [39] & 6 & 45 & 13,33 \\
\hline Bolu-Düzce [16] & 26 & 260 & 10,00 \\
\hline Kocaeli [33] & 17 & 79 & 21,52 \\
\hline Bartın-Küre [36] & 11 & 65 & 16,92 \\
\hline Karabük-Şeker Kanyonu [35] & 11 & 92 & 11,96 \\
\hline Kastamonu-Küre [38] & 5 & 41 & 12,20 \\
\hline Kastamonu-Ilgaz Dağ1 [3] & 21 & 203 & 10,34 \\
\hline
\end{tabular}

TEŞEKKÜR: Bu çalışmada bitki türlerinin tanımlanması Düzce Üniversitesi Orman Fakültesi'nin Orman Botaniği Anabilim Dalı öğretim üyeleri, Prof. Dr. Necmi AKSOY ve Dr. Öğr. Üyesi Turgay BİRTÜRK tarafından yapılmıştır.

\section{$\underline{\text { V. KAYNAKLAR }}$}

[1] H. H. Doğan, C. Öztürk, G. Kaşik, and S. Aktaş, "A checklist of aphyllophorales of Turkey,” Pakistan J. Bot., 2005.

[2] E. Sesli and C. M. Denchev, "Checklists of the myxomycetes, larger ascomycetes, and larger basidiomycetes in Turkey," Mycotaxon, 2008.

[3] Akata I., "Ilgaz Dağı Milli Parkı ve Yakın Çevresinin Makrofungus Florası,” Ankara Üniversitesi, 2010.

[4] M. G. Akata, I., Halıc1, “A New Lycoperdon record for Turkish Mycobiota," Mantar Derg., pp. 9-11, 2010.

[5] H. Kırış, Z., Halıcı, M.G., Akata, I., Allı, "Macrofungi of Akdağmadeni and Gemerek," Biodicon, c. 5, s . 2, ss. 53-58, 2012.

[6] K. Türkoğlu, A., Gezer, "Hacer Ormanı (Kayseri)'nın Makrofungusları," Ekoloji, c. 15, ss. 4348, 2006. 
[7] I. Akata and M. G. Halici, "Additional macrofungi records from Trabzon province for the mycobiota of Turkey,” Turk J Bot, 2011.

[8] H. All, "Macrofungi of Kemaliye district (Erzincan)," Turk. J. Bot., vol. 35, no. 3, pp. 299308, 2011.

[9] H. Alli, M. Isiloglu, and M. H. Solak, "New Ascomycete records for the macrofungi of Turkey," Turk. J. Botany, 2011.

[10] H. H. Doğan, M. A. Küçük, and I. Akata, "A Study on macrofungal diversity of bozyazi province (Mersin), Turkey,” Gazi Univ. J. Sci., 2010.

[11] M. Güngör, H., All1, H. ve Iş̧loğlu, “Ülkemiz Mikotasına İki Yeni Makrofungus Kayd1,” in IX. Türkiye Yemeklik Mantar Kongresi, Denizli, 2012, p. 76.

[12] H. H. Kaşık, G., Öztürk, C., Türkoğlu, A., Doğan, "Macrofungi flora of Yeşilhisar district (Kayseri)," Ot Sist. Bot. Derg., vol. 9, no. 2, pp. 123-134, 2002.

[13] H. H. Kaşık, G., Öztürk, C., Türkoğlu, A., Doğan, "Macrofungi of Yahyalı (Kayseri) Province," Turk. J. Botany, vol. 27, no. 6, pp. 453-462, 2003.

[14] A. Keleş and K. Demirel, "Macrofungal diversity of Erzincan province (Turkey)," Int. J. Bot., 2010.

[15] S. Sümer, "Batı Karadeniz Bölgesi, Özellikle Bolu Çevresinde Bulunan Odun Tahripçisi Mantarlar," İstanbul Üniversitesi Orman Fakültesi Yayınlarl, İ.Ü. Yayın No 2907, O.F. Yayın No 312, Istanbul, 1982.

[16] D. Yağiz, A. Afyon, M. Konuk, and S. Helfer, "Contributions to the macrofungi of Bolu and Düzce Provinces, Turkey," Mycotaxon. 2006.

[17] Y. . Yüksel, B., Akbulut, S., Baysal, İ., Gültekin, “Düzce yöresinin yenilebilir mantarlar1,” in $I$. Uluslarası Odun Dışı Orman Ürünleri Sempozyumu, 2007, p. 7.

[18] R. Watling, "Identification of The Larger Fungi," Hult. Educ. Publ. Ltd, p. 281, 1973.

[19] P. . Orton, "British Fungus Flora Agaricales and Boleti 4:," Pluteus \& Volvariella, p. 99, 1986.

[20] A. Caballero, "Lepiota cortinarius J.E. Lange, una rara especie encontrada en Lugo (Galicia, España)," Micolucus, N, pp. 8-12, 2015.

[21] J. F. Liang, "Taxonomy and phylogeny in Lepiota sect. Stenosporae from China," Mycologia, 2016.

[22] B. Pegler, D., Spooner, “The Mushroom Identifier,” in Grange Books, 1992, p. 144.

[23] E. . Vellinga, "No TitlePhylogeny of Lepiota (Agaricaceae)-Evidence from nrITS and nrLSU 
sequences," Mycol Prog, vol. 2, pp. 305-322, 2003.

[24] E. Peintner, U., Horak, "Lepiota and Cystolepiota (Agaricales) in Arctic-alpine habitats," Osterreisisches Zeitschrift fur Pilzkd., vol. 8, pp. 19-34, 1999.

[25] R. Tofts, “The British Species Of Lepiota 1: Section Stenosporae," F. Mycol., vol. 3, no. 4, pp. 124-136, 2002.

[26] M. Jordan, “The Encyclopedia of Fungi of Britain and Europe,” Fr. Lincoln, London., 2004.

[27] P. Leonard, "Lactarius Synoptic keys to British Species of Lactarius," BMS Keys, p. 27, 2008.

[28] P. Gierczyk, B., Kujawa, A., Szczepkowski, A., Chachuła, "Rare species of Lepiota and related genera.," Acta Mycol., vol. 46, pp. 137-178, 2011.

[29] P. Sysouphanthong, K. D. Hyde, E. Chukeatirote, and E. C. Vellinga, "A review of genus Lepiota and its distribution in east Asia," Curr. Res. Environ. Appl. Mycol., 2011.

[30] J. . Kirk, P.M., Cannon, P.F., Minter, D.W., Stalpers, "Ainsworth \& Bisby's Dictionary of the Fungi. 10th ed. Wallingford, UK," CABI Publ., p. 369, 2008.

[31] E. C. Vellinga, "Notulae ad floram agaricinam Neerlandicam - XVIII. Some notes on Cystolepiota and Lepiota," Publ. by Rijksherbarium/Hortus Bot. Leiden Persoonia, vol. 14, no. 4, pp. 407-415, 1992.

[32] H. Yilmaz and N. Zencirci, "Ethnomycology of Macrofungi in the Western Black Sea Region of Turkey: Identification to Marketing," Econ. Bot., 2016.

[33] A. Karakaya, "Kocaeli Yöresi Makrofunguslarının Belirlenmesi," Çevre ve Orman Bakanl. Yayın No 383, Çevre ve Orman Bakanl. Kavak ve Hızlı Gelişen Orman Ağaçları Araştırma Enstitüsü Müdürlüğü Yayın No 259, 2009.

[34] H. Servi, "Bolu Abant Tabiat Parkı makrofungus florası, Yüksek Lisans Tezi," Ankara Üniversitesi, 2010.

[35] B. Ç. Gültekin, "Şeker kanyonu (Yenice/Karabük) makrofungusları, Yüksek Lisans Tezi," Selçuk Üniversitesi, 2014.

[36] Y. Yeşilbaş, “Küre Dağları Milli Parkı'nın Bartın ili sınırlarında kalan bölümünün makrofungus floras1,Yüksek Lisans Tezi," Bartın Ünv., 2015.

[37] A. G. Şahin, U., Doğan, C., Baştar, F., Yılmaz, H., Yüksel, B., Lehtijarvi, A.T., Aday Kaya, "Bolu-Şerif Yüksel Araştırma Ormanında Bulunan Makrofunguslar ve Envanteri," Batı Karadeniz Orman. Araştırma Enstitüsü Müdürlüğü Yayını, Bolu, p. 57, 2016.

[38] M. Y. Özkazanç, N.Y., Oğuz, “Küre Dağları Milli Parkı'nın Kastamonu ili sınırlarında kalan bölümünün makrofunguslar1," Kastamonu Üniversitesi Orman Fakültesi Derg., vol. 17, no. 4, pp. 643-651, 2017. 
[39] Ç. Yalçın, M., Doğan, H.H., Akçay, "Identification of wood-decay fungi and assessment of damage in log depots of Western Black Sea Region (Turkey)," For. Pathol., pp. 1-12, 2019.

[40] P. V. Gassibe, J. A. Oria-de-Rueda, and P. Martín-Pinto, "P. pinaster under extreme ecological conditions provides high fungal production and diversity," For. Ecol. Manage., 2015. 